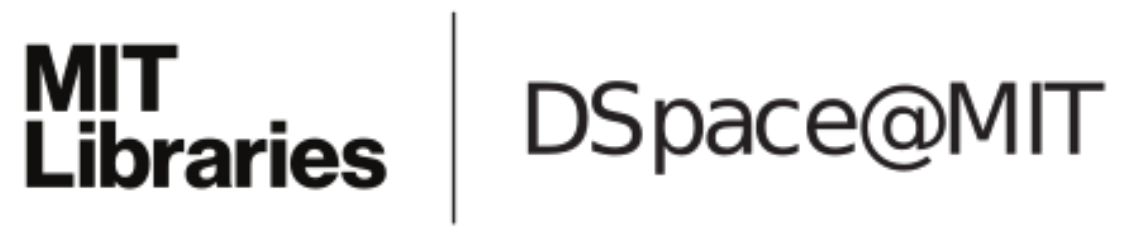

\author{
MIT Open Access Articles
}

Atom sieve for nanometer resolution neutral helium microscopy

The MIT Faculty has made this article openly available. Please share how this access benefits you. Your story matters.

Citation: Flatabø, Ranveig et al. "Atom sieve for nanometer resolution neutral helium microscopy." Journal of Vacuum Science and Technology B: Nanotechnology and Microelectronics 35, 6 (November 2016): 06G502 @ 2017 American Vacuum Society

As Published: http://dx.doi.org/10.1116/1.4994330

Publisher: American Vacuum Society

Persistent URL: https://hdl.handle.net/1721.1/126228

Version: Author's final manuscript: final author's manuscript post peer review, without publisher's formatting or copy editing

Terms of use: Creative Commons Attribution-Noncommercial-Share Alike 
Running title: Atom sieve for nanometer resolution neutral helium microscopy Running Authors: Flatabø et al.

\section{Atom sieve for nanometer resolution neutral helium microscopy}

Ranveig Flatab $\varnothing,{ }^{1,2, a)}$ Martin M. Greve, ${ }^{1}$ Sabrina D. Eder, ${ }^{1}$ Matthias Kalläne, ${ }^{3,4}$ Adrià Salvador Palau, ${ }^{5}$ Karl K. Berggren, ${ }^{2}$ and Bodil Holst ${ }^{1, b)}$

1) Department of Physics and Technology, University of Bergen, Allegaten 55, 5007 Bergen, Norway

2) Research Laboratory of Electronics, Massachusetts Institute of Technology, MA 02139, USA

3) Institute for Experimental and Applied Physics, University of Kiel, Leibnizstrasse 19, 24098 Kiel, Germany

4) Ruprecht Haensel Laboratory, University of Kiel and DESY, Kiel, Germany

5) Department of Engineering, Institute for Manufacturing, University of Cambridge, CB30FS, UK 
Neutral helium microscopy is a new tool for imaging fragile and/or insulating structures as well as structures with large aspect ratios. In one configuration of the microscope, the neutral helium atoms are focused as de Broglie matter waves using a Fresnel zone plate. The ultimate resolution is determined by the width of the outermost zone. Due to the low-energy beam (typically less than $0.1 \mathrm{eV}$ ), the neutral helium atoms do not penetrate solid material and the Fresnel zone plate therefore has to be a free-standing structure. This creates particular fabrication challenges. The so-called Fresnel photon sieve structure is especially attractive in this context, as it consists merely of holes. Holes are easier to fabricate than the free-standing rings required in a standard Fresnel zone plate for helium microscopy, and the diameter of the outermost holes can be larger than the width of the zone that they cover. Recently, a photon sieve structure was used for the first time, as an atom sieve, to focus a beam of helium atoms down to a few $\mu \mathrm{m}$. The holes were randomly distributed along the Fresnel zones to suppress higher order foci and side lobes. Here we present a new atom sieve design with holes distributed along the Fresnel zones with a fixed gap. This design gives higher transmission and higher intensity in the first order focus. We present an alternative electron beam lithography fabrication procedure that can be used for making such high transmission atom sieves with very high resolution, potentially smaller than $10 \mathrm{~nm}$. The atom sieves were patterned on a $35 \mathrm{~nm}$ or a $50 \mathrm{~nm}$ thick silicon nitride membrane. The smallest hole is $35 \mathrm{~nm}$, the largest $376 \mathrm{~nm}$. In a separate experiment, patterning $\mu \mathrm{m}$-scale areas with hole sizes down to $15 \mathrm{~nm}$ is demonstrated. The smallest gap between neighbouring holes in the atom sieves is $40 \mathrm{~nm}$. They have 47011 holes each and are $23.58 \mu \mathrm{m}$ in diameter. The opening ratio is $22.60 \%$, and the Fresnel zone coverage of the innermost zones is as high as 0.68 . This high-density pattern comes with certain fabrication challenges, which we discuss.

a) Electronic mail: ranveig.flatabo@uib.no

b) Electronic mail: bodil.holst@uib.no 


\section{INTRODUCTION}

The first neutral helium microscopy images were published in $2008^{1}$. Several groups have worked on the technique ${ }^{2-6}$. In a neutral helium microscope (NEMI), a beam of neutral helium atoms with narrow velocity (wavelength) distribution is used to image a sample. An image is obtained by measuring either the reflected or transmitted signal as the beam is scanned across the sample. The energy of the beam is less than $0.1 \mathrm{eV}$ for a wavelength of $0.1 \mathrm{~nm}$, which means that the atoms probe the outermost electron density distribution of the sample without penetrating into solid material ${ }^{7}$. These properties make neutral helium microscopy particularly suited for the investigation of fragile and/or insulating surfaces as well as high aspect ratio structures. Recently it was shown that helium microscopy can distinguish between different metal films on an insulating substrate ${ }^{5}$. However, the big challenge is to create a high-resolution microscope, partly because the focusing element must be of the binary type with either completely transparent or completely opaque areas.

Helium-atom focusing with a binary Fresnel zone plate was first carried out by Carnal et al. using a beam of metastable helium atoms ${ }^{8}$. The first focusing of a neutral, ground state helium beam with a zone plate was carried out by Doak et al ${ }^{9,11}$. Currently, the best zone plates made for helium microscopy have a nominal outermost zone width of $50 \mathrm{~nm}^{10}$.

The Beynon-Gabor zone plate, which is also binary, has been suggested as an alternative candidate for focusing helium atoms ${ }^{12}$. The main advantage of the Beynon-Gabor zone plate is that it has no higher-order foci. Unfortunately, it is very challenging to fabricate. In 2015 a new optical diffraction element, the atom sieve, was introduced and used to focus helium atoms down to $3.6 \mu \mathrm{m}^{13}$. The atom sieve is a direct analog to the photon sieve, which was invented in 2001 for applications with soft X-rays ${ }^{14}$. It consists of holes of varying diameter, arranged across the Fresnel zones in such a manner that it is possible to focus to a spot with a diameter smaller than the smallest hole. In addition, higher-order diffraction and secondary maxima can be suppressed. There are several advantages of the atom sieve. Firstly, it is easier to fabricate free-standing holes than free-standing ring segments as no support structure is needed. Moreover, the design can be tuned so that the diameter of the outermost hole is larger than the width of the outermost zone that it covers. In contrast, 
the minimum structure size of a first order Fresnel zone plate is limited by the width of the outermost zone ${ }^{27}$. A higher resolution can be obtained by using higher order foci, but this comes with a significant reduction in intensity. For a standard Fresnel zone plate $10.1 \%$ of the incident beam goes into the first order focus and only $1.1 \%$ into the third order ${ }^{17}$. For an atom Fresnel zoneplate these numbers are further reduced due to the support rods needed to keep the zone plate ring structure together. Intensity is a big issue in helium microscopy ${ }^{15,16}$, so this is a major limitation.

Here we present a new high-transmission atom sieve design. The paper begins with an experimental section, where we first present the design requirements followed by the fabrication procedure. There then follows a result and discussion section showing SEM images of the fabricated atom sieves and the first atom sieve transmission tests demonstrating that the structure is transparent to atoms. The paper finishes with a summary and conclusion.

\section{EXPERIMENTAL}

\section{A. Atom Sieve Design Considerations}

In the original photon sieve work, suppression of higher order foci and side lobes were particularly important, and a Weber transmission window was used, giving holes randomly distributed along the zones ${ }^{14}$. This limits the number of holes and hence the open area ratio (total transmissivity). The first atom sieve used the original photon sieve design and had an opening ratio (total transmissivity) of $9.22 \%$ only, giving a transmissivity of $1.86 \%$ of the incident beam into the first order focus ${ }^{13}$. For the neutral helium microscope it is crucial to ensure maximum intensity in the first order focus, as discussed in the introduction. For this reason we changed the design of the atom sieve, and used a transmission window similar to a rectangular shaped window, but with increasing transmission towards the center, to fit as many holes as possible in each zone based on a predefined minimum gap. The minimum gap is discussed below. By doing so, the opening ratio of the sieve (total transmissivity) increases to $22.60 \%$ and the intensity in the first order focus is $4.57 \%$.

Another important decision was to determine hole sizes that could realistically be patterned. This is important for the resolution, but also for the transmissivity, as it determines how large a zone plate can be made for a given focal length. The focal length was chosen so 
that it corresponds to a microscope that can be constructed and further so that the velocity spread of the beam does not cause chromatic abberations (see Ref. 15). The hole size varies from $376 \mathrm{~nm}$ to $35 \mathrm{~nm}$. The smallest holes that have ever been made with direct-write lithography are around $1 \mathrm{~nm}$ in size. They were made using helium ion beam lithography ${ }^{18}$. Direct-write ion lithography is in principle an attractive technique for atom sieve patterns, as one can pattern directly on the membrane i.e. no resist is needed. This enables a one-step fabrication process where the result can be examined in-situ. Moreover, the backscattering contribution to the lithographic point spread function of light ions, such as helium and neon, is shown to be minimal, and hence dose modifications might not be needed ${ }^{19,20}$. However, these are relatively new techniques with low throughput, limited stability and, in the case of helium ions, low sputtering yields ${ }^{21}$. Electron beam lithography, followed by reactive ion etching, was chosen as the method of fabrication, and an atom sieve pattern with a minimum hole size of $35 \mathrm{~nm}$ was realized. To ensure maximum transmissivity the diameter of the holes was chosen to be the same as the zone widths. As discussed in the introduction, it is possible to design a transmission function enabling the hole diameter to be larger than its zone, but this comes at the cost of intensity.

The aim is eventually to install the atom sieve in a new helium microscope, currently being designed. This determined a focal length of approximately $10 \mathrm{~mm}$ for a helium atom de Broglie wavelength of approximately $0.1 \mathrm{~nm}$ for the sieve. With minimum hole size and focal length given, the sieve diameter could be calculated (the sieve diameter is $23.58 \mu \mathrm{m}$ ).

Finally, the gap between adjacent holes was selected. This is essential for the transmission of the device, since large values reduce the number of holes. The gap between neighbouring holes varies from $53 \mathrm{~nm}$ to $40 \mathrm{~nm}$, depending on how many holes one can fit into one zone. Hence, the zones in the atom sieve have a very high zone coverage (i.e. exposed area in one Fresnel zone divided by the total area of the corresponding zone). The zone coverage of the innermost zone is 0.68 (corresponding to a hole diameter of $376 \mathrm{~nm}$ and a gap of $53 \mathrm{~nm}$ ) and it slowly decreases to 0.39 for the outermost zone (for a $35 \mathrm{~nm}$ Fresnel zone overlaid with holes of $35 \mathrm{~nm}$ and a gap of $40 \mathrm{~nm}$ ). The minimum gap of $40 \mathrm{~nm}$ was chosen as a safe value, making sure that it was possible to pattern separate holes with electron beam lithography and transfer the pattern into the membrane.

The atom sieve design is shown in Fig. 1. Note that it is not rotationally symmetrical. The stripe in the horizontal direction exists as the positioning of the holes start at the same 
angle on each zone. Each zone is filled with as many holes as possible (with the given minimum hole distance).

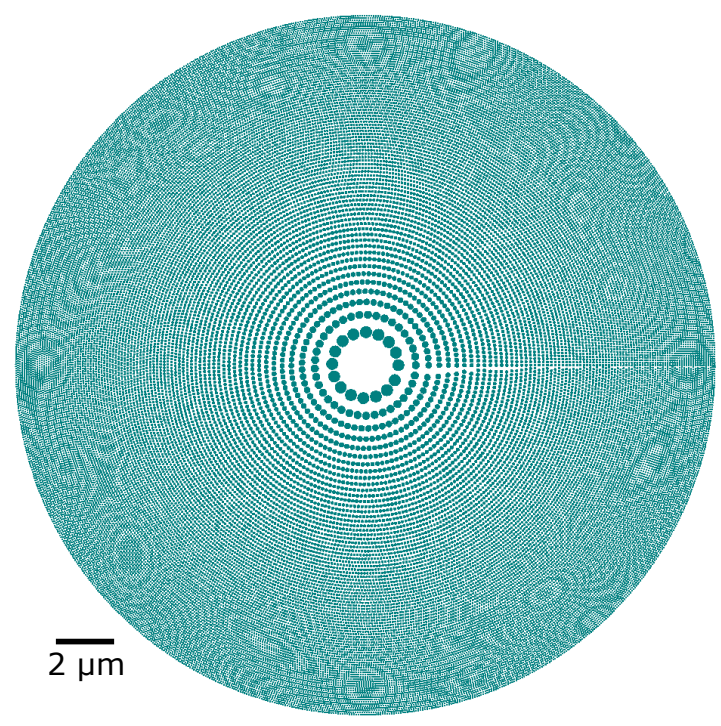

FIG. 1. Atom sieve design maximized for total transmissivity. The pattern is not symmetrical, which is discussed in the text. The central opaque area corresponds to the first zone, which is kept closed for stability reasons.

\section{B. Fabrication Procedure}

The fabrication process is illustrated in Fig. 2. It shares some steps with the fabrication procedure used $\mathrm{in}^{22}$, but with some modifications. In this work we use $35 \mathrm{~nm}$ or $50 \mathrm{~nm}$ thick SiNx membranes with low stress (low stress membrane was chosen to prevent the membrane from cracking). The membrane thicknesses were chosen to keep the aspect ratio of the etched holes adequate. However, it comes with the obvious trade-off of being more fragile than thicker membranes, which we discuss in Sec. III.

First, photolithography was used to pattern membrane windows on the backside of the $\mathrm{SiNx} / \mathrm{Si} / \mathrm{SiNx}$ wafer. To open the membrane windows, a dry etch step was performed using 15 SCCM (gas flow rate: standard cubic centimeters per minute) $\mathrm{CF}_{4}, 150 \mathrm{~W}, 10$ mTorr for 2 minutes. Then, the underlying silicon was etched using $\mathrm{KOH}(30 \% \mathrm{KOH}$ in de-ionized water) at $80^{\circ} \mathrm{C}$, creating $\mathrm{SiNx}$ membranes. 


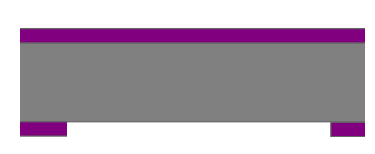

Step 1:

Open membrane backside using optical lithography

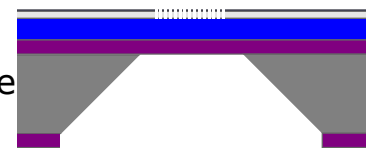

RIE step 1:

Transfer pattern into $\mathrm{SiO}_{x}$ using CF4 dry etch

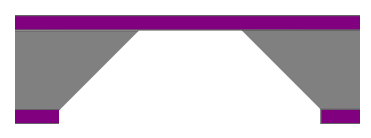

Step 2:

$\mathrm{KOH}$ etch to create

freestanding membrane

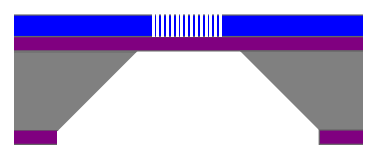

RIE step 2:

Transfer pattern into ARC using $\mathrm{O}_{2} / \mathrm{He}$ dry etch
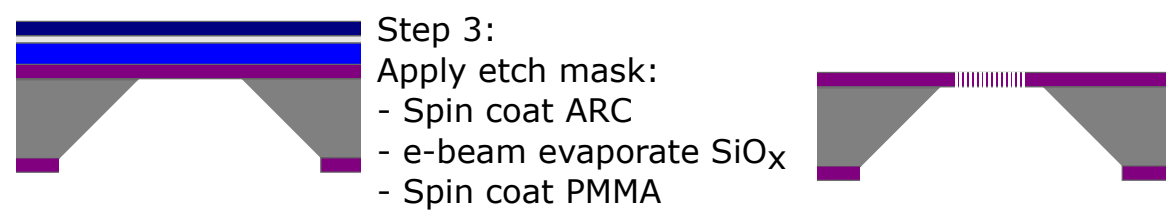

RIE step 3:

Transfer pattern into $\mathrm{SiN}_{\mathrm{X}}$ using $\mathrm{CF}_{4}$ dry etch

$\operatorname{SiN}_{x}$

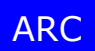

Si

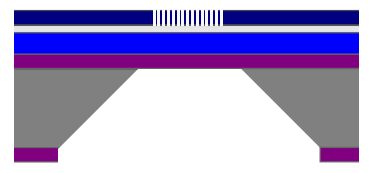

Expose and develop PMMA

$\mathrm{SiO}_{2}$

PMMA

FIG. 2. (Color online)Step-by-step fabrication procedure for creating the free-standing membrane structure.

As the resist, poly(methylmethacrylate) (PMMA) alone cannot withstand the reactive ion etching that creates free-standing holes, the membrane was coated with an etch mask consisting of $110 \mathrm{~nm}$ antireflective coating (ARC), $10 \mathrm{~nm} \mathrm{SiO}_{\mathrm{x}}$ and $60 \mathrm{~nm} \mathrm{PMMA} \mathrm{(2 \%} \mathrm{in}$ anisole, 950 K). Firstly, ARC was spin-coated onto the chip at 3000 RPM for 1 minute and baked at $180^{\circ} \mathrm{C}$ for 3 minutes. $10 \mathrm{~nm} \mathrm{SiO}_{\mathrm{x}}$ was then deposited by the means of electron beam evaporation. Finally, PMMA was spin-coated onto the sample at 3000 RPM for 1 minute and baked at $180^{\circ} \mathrm{C}$ for 3 minutes.

The PMMA was exposed using $125 \mathrm{keV}$ electrons (ELS-F125 Elionix), $1 \mathrm{nA}$ current and $2.5 \mathrm{~nm}$ step size. As the beam enters the resist, the electrons interact with the resist and the substrate and causes energy to be delivered to points different from the point of incidence, commonly known as proximity effects. Due to the high zone coverage of the atom sieve design (0.68 to 0.39$)$ it is extremely sensitive to such unintended exposure, especially as the final structure needs to be free-standing. To find the correct dose (i.e. areal dose density) 
multiple atom sieves were patterned with a single exposure dose, analogous to the standard dose matrices. The innermost zones are receiving the largest dose per zone and get the largest amount of unintended exposure. This causes over-exposure. Therefore, the dose was linearly increased from $2.8 \mathrm{mC} / \mathrm{cm}^{2}$ to $3.8 \mathrm{mC} / \mathrm{cm}^{2}$ going from the innermost zone to the outermost zone (again using a $125 \mathrm{keV}$ electron beam, $1 \mathrm{nA}$ current and $2.5 \mathrm{~nm}$ step size). It should be noted that there are accurate and rigorous ways to calculate the correct dose needed in a pattern of high surface coverage ${ }^{23,28,29}$. However, as a crucial part in this work is to transfer the pattern into the membrane, which also causes a minor hole broadening (discussed below), the correct doses was found by iteration.

The sample was developed in 1:3 MIBK:IPA (methyl isobutyl ketone:2-propanol)at $0^{\circ} \mathrm{C}$ for 30 seconds, and dried using pressurized nitrogen. Cold development was chosen, as it has been found to improve feature quality and resolution ${ }^{24,25}$.

The pattern was transferred to the $\mathrm{SiO}_{\mathrm{x}}$-layer using $15 \mathrm{SCCM}$ of $\mathrm{CF}_{4}$ at $150 \mathrm{~W}$ and 10 mTorr. Pattern transfer into the ARC was done using 20 SCCM He and 10 SCCM of $\mathrm{O}_{2}$ at 10 mTorr and 200 W. Finally, pattern transfer into the SiNx was done using 15 SCCM of $\mathrm{CF}_{4}$ at $150 \mathrm{~W}$ and 10 mTorr. Reactive ion etching produces anisotropic etch profiles. Nevertheless, a broadening of about $5 \mathrm{~nm}$ in the diameter is found when comparing circles in an atom sieve that was not etched (i.e. atom sieve in PMMA) with holes in an atom sieves.

\section{RESULTS AND DISCUSSION}

\section{A. The Atom Sieve}

Figure 3 shows an overview image of an atom sieve. The diameter of the sieve is $23.58 \mu \mathrm{m}$ $\pm 0.01 \mu \mathrm{m}$ and it contains 47011 holes. Close-up SEM images are given in Fig. 4. The holes of the innermost zone have a diameter of $378 \mathrm{~nm} \pm 4 \mathrm{~nm}$, and the holes of the outermost zone $38 \mathrm{~nm} \pm 4 \mathrm{~nm}$. Both slightly too large and slightly too small holes will lead to a reduction in transmissivity into the first order focus, because a too large hole will open up into the neighbouring zone and give destructive interference. The overall deviation, taken for all holes is so small that the reduction is likely to be negligible ${ }^{30}$. It is very important for the function of the atom sieve that the size of the holes and their position are correct 
to within a fraction of the Fresnel zone they cover, since otherwise a hole will contribute with destructive interference ${ }^{30}$. In other words, the positioning of the small holes needs to be more accurate than the positioning of the big holes. In Fig. 5 a SEM image of the fabricated atom sieve (black holes) is superimposed with the design pattern (white "holes"). As can be seen, the positioning of even the smallest holes is accurate to within a fraction of the hole diameter.

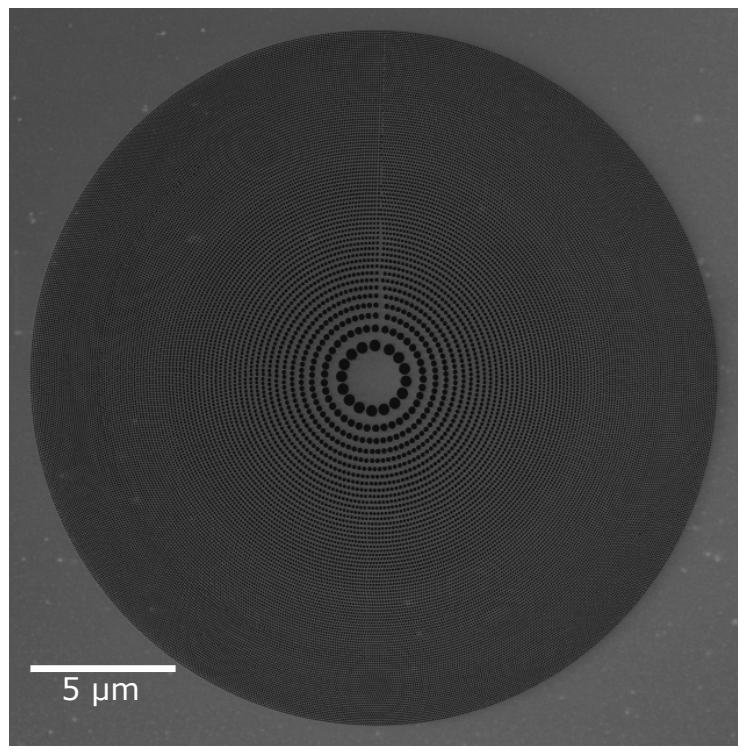

FIG. 3. Overview SEM image of the fabricated free-standing atom sieve.

The membrane thickness of $35 \mathrm{~nm}$ was chosen to keep the aspect ratio of the etched holes adequate. However, this comes with a trade-off of being more fragile than a thicker membrane. The 35-nm thick membranes easily broke due to handling or transport. An improvement in stability was seen when using a membrane thickness of $50 \mathrm{~nm}$.

In a separate experiment it was desirable to investigate the smallest diameter hole-sizes. A lower hole density, and a random hole distribution was selected for these test experiments, as illustrated in Fig. 6. The diameter of the holes in the SEM image ranges from $25 \mathrm{~nm}$ to $15 \mathrm{~nm}$. 


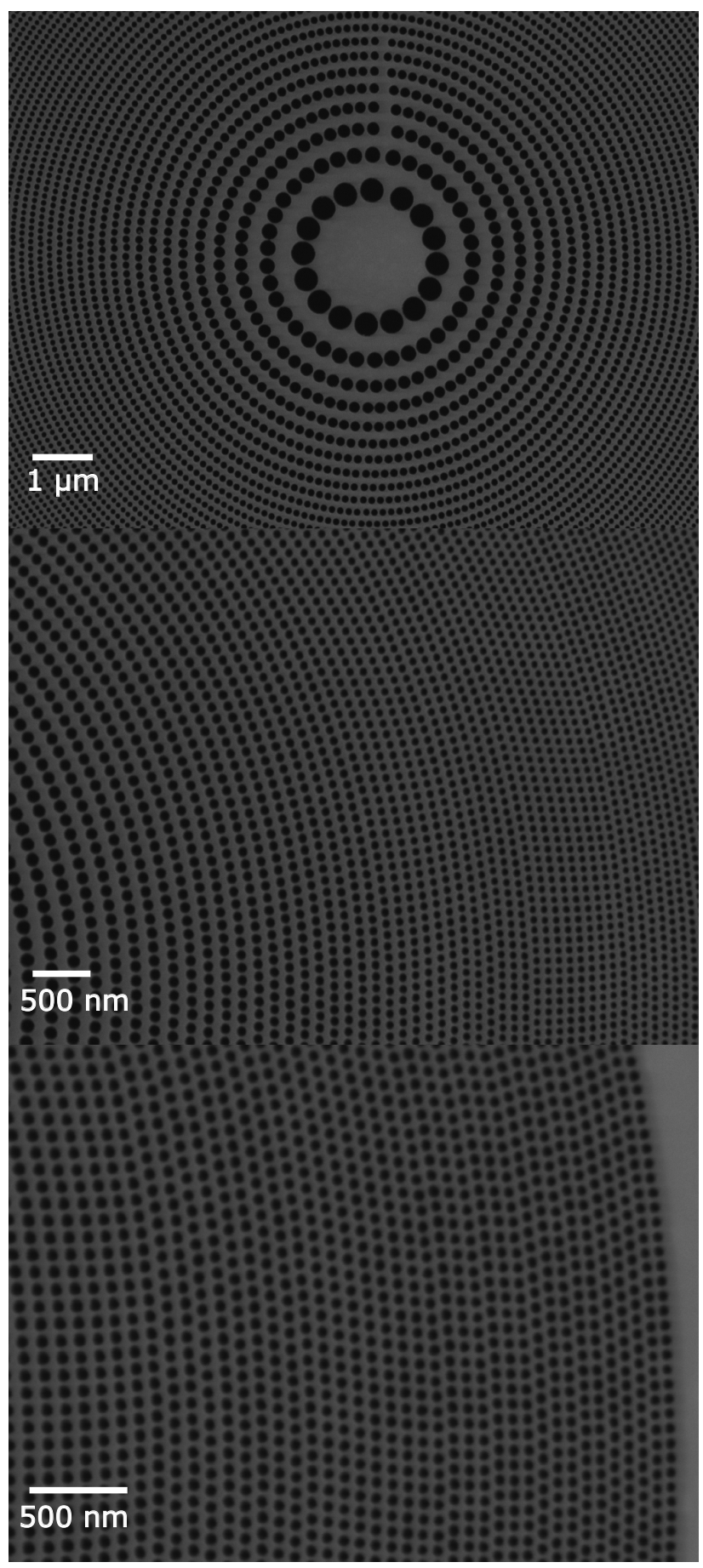

FIG. 4. Close-up SEM images of a free-standing atom sieve. The holes of the innermost zone have a diameter of $378 \mathrm{~nm} \pm 4 \mathrm{~nm}$, and the holes of the outermost zone are found to be $38 \mathrm{~nm} \pm 4 \mathrm{~nm}$. 


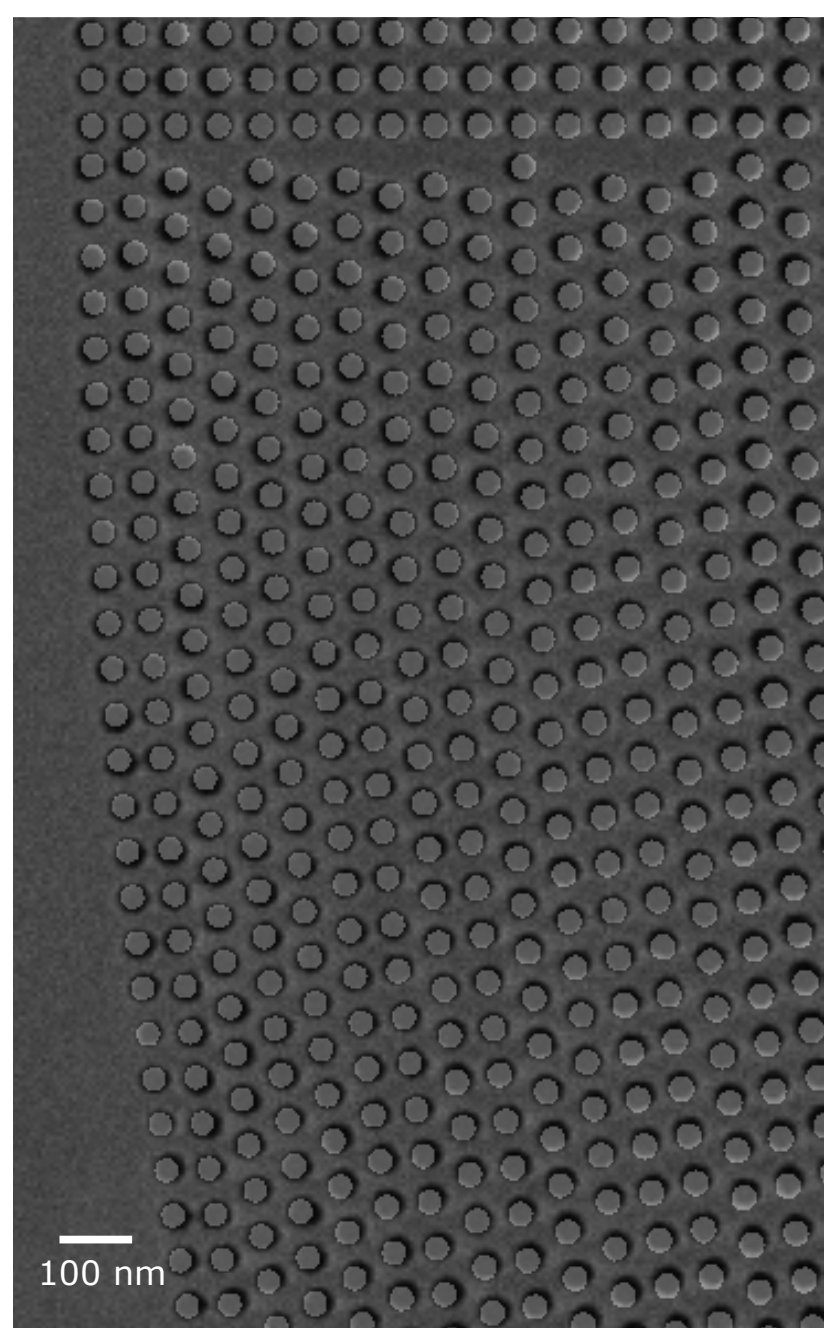

FIG. 5. Overview SEM image of the free-standing atom sieve where the design pattern is superimposed onto the image. The design-holes are white, while the underlying SEM image consists of black holes.

\section{B. Atom Transmission Tests}

Ultimately the atom sieve will be integrated in a new neutral helium atom microscope instrument. However, prior to that we wanted to check if it is transmissive to atoms. This is crucial, because in order to be a functional atom sieve, the holes must be free standing. It can be difficult to evaluate just by optical inspection or inspection in a SEM if all material has been removed. This is illustrated in Fig. 7, where a $20 \mu \mathrm{m}$ circle on a SiNx membrane appears to be fully etched, but in fact is not. For this reason we installed the atom sieve as a sample in our existing helium microscope ${ }^{26}$ and measured the overall transmissivity by 


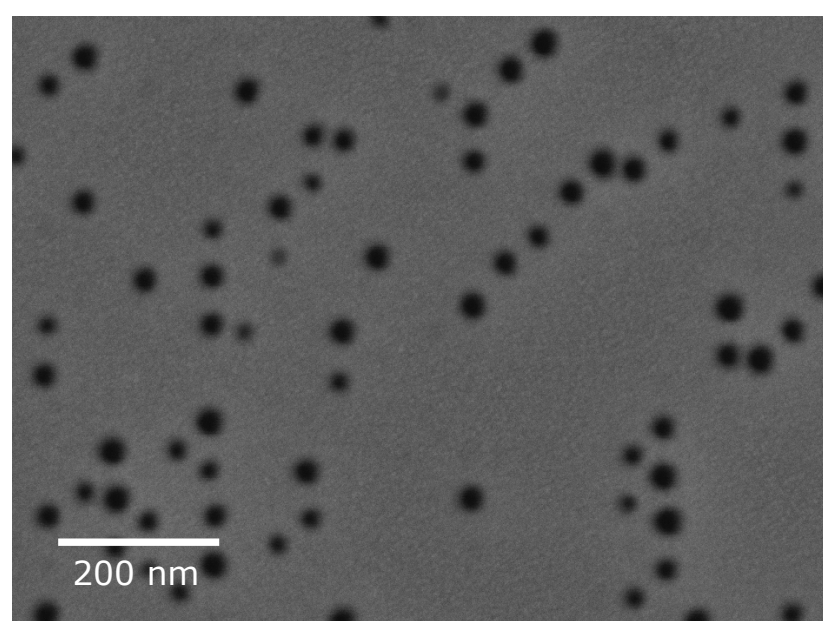

FIG. 6. Free-standing holes with a diameter ranging from $25 \mathrm{~nm}$ to $15 \mathrm{~nm}$.

sending a $20 \mu \mathrm{m}$ helium beam onto the sample. Presently the minimum resolution in our helium microscope is only around $5 \mu \mathrm{m}$, so it was not possible to do a detailed mapping. Figure 8 presents a line scan of the sieve. The transmissivity is normalized to the intensity measured when no sample is present (i.e. $100 \%$ signal), and the background has been subtracted. The transmissivity of the sieve is found to be $23.8 \% \pm 3 \%$, in good agreement with the nominal transmissivity of $22.6 \%$.

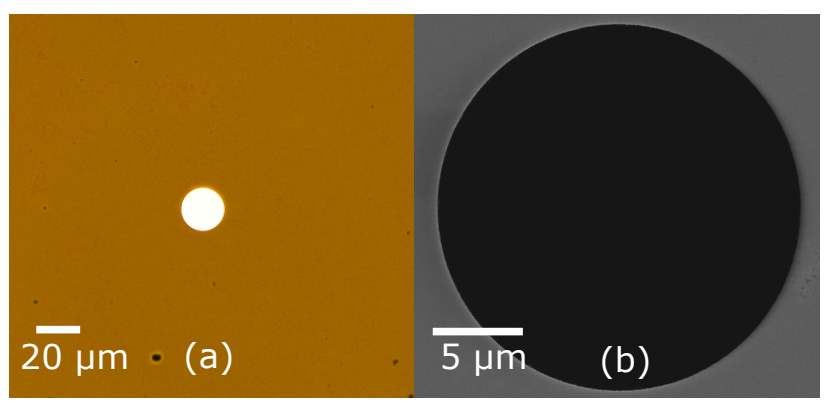

FIG. 7. (a) Transmission optical microscopy image and (b) SEM image of a $20 \mu \mathrm{m}$ circle in a 200 nm thick SiNx membrane that appears to be free-standing. However, transmission helium atom microscopy measurements revealed that the structure was not etched through. 


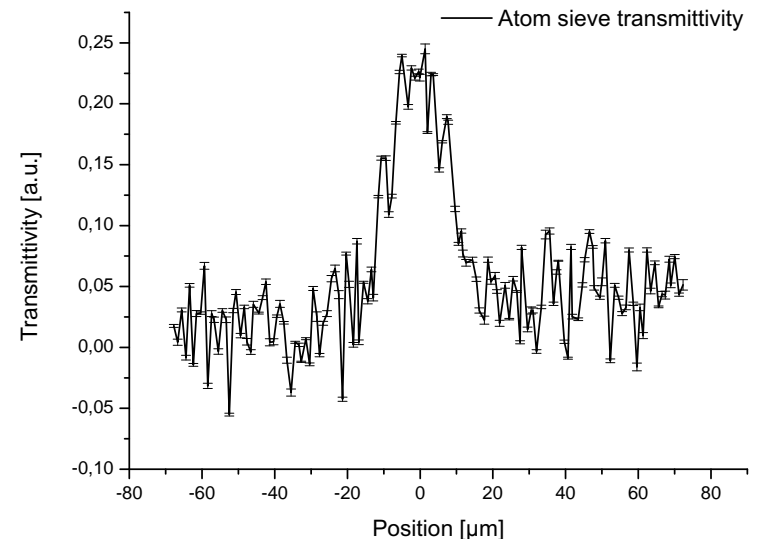

FIG. 8. Overall transmissivity of the atom sieve measured in the neutral helium microscope. The transmissivity of the sieve is found to be $23.8 \% \pm 3 \%$, in good agreement with the nominal transmissivity of $22.6 \%$.

\section{SUMMARY AND CONCLUSION}

We have fabricated atom sieves, with a smallest hole diameter of $35 \mathrm{~nm}$. The atom sieves were designed for maximum transmission so that the diameter of the smallest hole corresponds to the width of the outermost zone. This means that we have made a zone plate which can be used to do neutral helium microscopy with resolution in the range of 35 nm. In addition, we have demonstrated writing of $\mu \mathrm{m}$-scale areas with holes down to 15 $\mathrm{nm}$ in diameter. By using a transmission window where the hole areas are reduced in the outer parts e.g. a Weber window, it is possible to design an atom sieve with a resolution larger than the hole diameter. In a recent paper Palau et $\mathrm{al}^{16}$ shows that with the velocity spread and intensity of present day beam sources and present day detector technology, the limiting factor for a realistic helium microscope design is the resolution of the optical element, determined by the width of the outermost zone. Thus our work shows that helium microscopy with a resolution better than $15 \mathrm{~nm}$ should be possible.

\section{ACKNOWLEDGEMENTS}

The authors thank M. Mondol, T. Savas and J. Daley at MIT Nanostructure Laboratory, and B. Samelin at University of Bergen Nanophysics Laboratory. This work was done at the MIT Nanostructure Laboratory and the MIT Scanning-Electron-Beam Lithography 
facility. The helium microscopy test was done at the University of Bergen, Nanophysics Laboratory. R. Flatabø gratefully acknowledges support from Bergen Research Foundation and the Norway-America Association through American-Scandinavian Foundation's The Stolt-Nilsen Fund for education. 


\section{REFERENCES}

${ }^{1}$ M. Koch, S. Rehbein, G. Schmahl, T. Reisinger, G. Bracco, W.E. Ernst and B. Holst, J. Microscop, 229, 1, 1, 2008

${ }^{2}$ P. Witham and E. Sánchez, Rev. Sci. Instrum., 82, 103705, 2011

${ }^{3}$ P. Witham and E. Sánchez, Cryst. Res. Technol., 49, 690, 2014

${ }^{4}$ A. Fahy, M. Barr, J. Martens, and P. Dastoor, Rev. Sci. Instrum. 86, 023704, 2015

${ }^{5}$ M. Barr, A. Fahy, J. Martens, A.P. Jardine, D.J. Ward, J. Ellis, W. Allison, and P.C. Dastoor, Nat. Commun. 7, 10189, 2016

${ }^{6}$ P. Witham and E. Sánchez, J. Microscop. 248, 223, 2012

${ }^{7}$ G. Bracco and B. Holst, Surface Science Techniques, (Springer Science \& Business Media), 2013

${ }^{8}$ O. Carnal, M. Sigel, T. Sleator, H. Takuma, and J. Mlynek, Phys. Rev. Lett. 67, 3231, 991

${ }^{9}$ R.B. Doak, R.E. Grisenti, S. Rehbein, G. Schmahl, J.P. Toennies, and C. Wöll, Phys. Rev. Lett. 83, 4229, 1999

${ }^{10}$ T. Reisinger, S. Eder, M.M. Greve, H.I. Smith, B. Holst, Microelectron. Eng., 87, 1011, 2010

${ }^{11}$ R. Rehbein, J. Phys. IV France, 104, 207, 2003

${ }^{12}$ M.M. Greve, A. Vial, J. Stamnes, and B. Holst, Opt. Express 21, 28483, 2013

${ }^{13}$ S.D. Eder, X. Guo, T. Kaltenbacher, M.M. Greve, M. Kalläne, L. Kipp, and B. Holst, Phys. Rev. A, 91, 043608, 2015

${ }^{14}$ L. Kipp, M. Skibowski, R.L. Johnson, R. Berndt, R. Adelung, S. Harm, and R. Seemann, Nature, 414, 184, 2002

${ }^{15}$ A.S. Palau, G. Bracco and B. Holst, Phys. Rev A, 95, 013611, 2017

${ }^{16}$ A.S. Palau, G. Bracco and B. Holst, Phys. Rev A, 94, 063624, 2016

${ }^{17}$ A.G. Michette, Optical Systems for Soft X Rays, (Plenum Press, New York), 1986

${ }^{18}$ D.A. Oulianov, R.A. Crowell, D.J. Gosztola, I.A. Shkrob, O.J. Korovyanko, and R.C. Rey-de-Castro, J. Appl. Phys. 101, 053102, 2007

${ }^{19}$ D. Winston, B.M. Cord, B. Ming, D.C. Bell, W.F. DiNatale, L.A. Stern, A.E. Vladar, M.T. Postek, M.K. Mondol, J.K.W. Yang, K.K. Berggren, J. Vac. Sci. Technol. B, 27, 2702,2009 
${ }^{20}$ D. Winston, V.R. Manfrinato, S.M. Nicaise, L.L Cheong, H. Duan, D. Ferranti, J. Marshman, S. McVey, L.A. Stern, J. Notte, K.K. Berggren, Nano Lett., 11, 4343, 2011

${ }^{21}$ M.M. Marshall, J. Yang, A.R Hall, Scanning, 34, 101, 2012

${ }^{22}$ J.O. Grepstad, M.M. Greve, T. Reisinger and B. Holst, J. Vac. Sci. Technol. B, 31, 06F402, 2013

${ }^{23}$ G. Owen, J. Vac. Sci. Technol. B, 8, 1889, 1990

${ }^{24}$ W. Hu, K. Sarveswaran, M. Lieberman, G.H. Bernstein, J. Vac. Sci. Technol. B, 22, 1711, 2004

${ }^{25}$ B. Cord, J. Lutkenhaus and K.K. Berggren, J. Vac. Sci. Technol. B, 25, 2013, 2007

${ }^{26}$ S.D. Eder, A.K. Ravn, B. Samelin, G. Bracco, A.S. Palau, T. Reisinger, E.B. Knudsen, K. Lefmann and B. Holst, Phys. Rev. A, 95, 023618, 2017

${ }^{27}$ G. Schmahl and D. Rudolph, Zone Plates for X-Ray Microscopy in X-Ray Microscopy. (Springer Series in Optical Sciences), 43, 1984

${ }^{28}$ M. Peckerar, R. Bass, K.W. Rhee, J. Vac. Sci. Technol. B, 18, 3143, 2000

${ }^{29}$ M. Parikh, Journ. Appl. Phys., 50, 4371, 1979

${ }^{30}$ E. Hecht, Optics 4 Edition, (Addison-Wesley), 2001 
